\title{
This Issue of Geochemistry International is Devoted to Studies of the Chelyabinsk Meteorite, Which Fell on February 15, 2013, Near the Town of Chelyabinsk
}

DOI: $10.1134 / \mathrm{S} 0016702913070161$

The Vernadsky Institute of Geochemistry and Analytical Chemistry, Russian Academy of Sciences, and the Committee on Meteorites of the Russian Academy of Sciences sent a search team to the meteorite impact site to collect samples of its material. Within a few days, meteorite fragments found in the area were delivered to laboratories. Small fragments (ranging from a few fractions of a centimeter to a few centimeters) penetrated the snow cover, which was $60-80 \mathrm{~cm}$ thick in the search area, and were almost completely uncontaminated.

Further petrological and geochemical analysis of the samples at the Vernadsky Institute has shown that the Chelyabinsk meteorite is an aerolite, carbonaceous chondrite, which was then categorized as LL5 based on its chemical and mineralogical characteristics and structure.

A portion of the collected meteorite material was also delivered to the Open University in England, where C. Pillinger and his colleagues examined the oxygen (three isotopes), nitrogen, and carbon isotopic compositions. Some papers published in this issue report the newly measured magnetic characteristics of the meteorite and the composition of its minerals. The paper by V.E. Fortov et al. is devoted to aerodynamic processes that accompanied the meteorite's entry into the Earth's atmosphere and their relationships with the strength characteristics of this meteorite.

The results of the studies published in this issue mark a new in vivo approach to studying meteorites, in which the compositional, dynamic, and astronomical aspects are studied in mutual relations.

Director of the Vernadsky Institute of Geochemistry and Analytical Chemistry, Russian Academy of Sciences, and the Chairman of the Committee on Meteorites, Russian Academy of Sciences, Academician E. M. Galimov

Translated by E. Kurdyukov 